\title{
Ganglion Cell Complex Parameters in Primary Angle Closure Suspects
}

\author{
Yizhen Tang a,b,c Xiaohua Pan ${ }^{d}$ Kai Cao $^{e}$ Hui Feng ${ }^{d}$ Yue Yang ${ }^{f}$ \\ Zhongyin $\mathrm{Hu}^{\mathrm{g}}$ Fancheng Yan ${ }^{\mathrm{h}}$ Ying Han ${ }^{\mathrm{i}}$ Shuning $\mathrm{Li}^{\mathrm{d}}$
}

aEye Institute and Department of Ophthalmology, Eye \& ENT Hospital, Fudan University, Shanghai, China; ${ }^{\text {NNHC }}$ Key Laboratory of Myopia (Fudan University), Key Laboratory of Myopia, Chinese Academy of Medical Sciences, Shanghai, China; 'Shanghai Key Laboratory of Visual Impairment and Restoration, Shanghai, China; ${ }^{d}$ Beijing Tongren Eye Center, Beijing Tongren Hospital, Capital Medical University, Beijing Ophthalmology and Visual

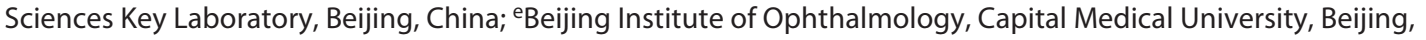
China; ${ }^{f}$ Department of Ophthalmology, The Third Hospital of Mianyang, Sichuan Mental Health Center, Mianyang, China; ${ }^{9}$ Department of Ophthalmology, First People's Hospital of Yunnan Province, Kunming, China; ${ }^{\text {h} D e p a r t m e n t ~}$ of Ophthalmology, Beijing Friendship Hospital, Capital Medical University, Beijing, China; 'Department of Ophthalmology, University of California San Francisco, San Francisco, CA, USA

\section{Keywords}

Primary angle closure suspects · Ganglion cell complex ·

Related factors · Biometry

\begin{abstract}
Introduction: This study aimed to investigate the ganglion cell complex (GCC) parameters in primary angle closure suspects (PACS) and identify the related factors. Methods: A total of 731 subjects, including 289 subjects with PACS and 442 subjects without PACS, underwent RTVue XR OCT. GCC parameters were compared between the two groups. The linear mixed-effects model was performed to evaluate the relationships between the GCC parameters and related factors. Results: Significant differences were found in gender, age, spherical refractive error, height, waist, anterior chamber depth, lens thickness, axial length, superior GCC thickness, ganglion cell complex focal loss volume, ganglion cell complex global loss volume, and ganglion cell complex root mean square between PACS and normal controls. The linear mixed-effects model showed that age $(p=0.008)$ and blood glucose $(p=0.001)$ were negatively correlated with average
\end{abstract}

GCC thickness in PACS subjects, and PACS $(p=0.036)$ and age $(p<0.001)$ were the key influencing factors for average GCC thickness. Conclusion: GCC parameters in PACS subjects are different from those in normal controls. Careful explanation should be considered when evaluating changes of GCC parameters in patients with PACD.

(c) 2021 The Author(s)

Published by S. Karger AG, Basel

\section{Introduction}

Primary angle closure suspect (PACS) is a subtype of primary angle closure disease (PACD) which has a high prevalence in Asia and leads to nearly half of the glaucomatous vision loss worldwide [1-3]. Evidence showed that $22 \%$ of PACS developed into PAC in 5-year followup [4]. Early intervention of PACS may prevent PACS from its progression [5-7]. However, it is unclear how subjects with PACS have progressed and when and how

Yizhen Tang and Xiaohua Pan are listed as co-first authors who contributed equally to the manuscript.
C 2021 The Author(s)

Published by S. Karger AG, Basel

This is an Open Access article licensed under the Creative Commons Attribution-NonCommercial-4.0 International License (CC BY-NC) (http://www.karger.com/Services/OpenAccessLicense), applicable to the online version of the article only. Usage and distribution for commercial purposes requires written permission.
Correspondence to:

Shuning Li, lishuningqd@ 163.com 
the retinal structure, such as retinal ganglion cells (RGCs) and retinal nerve fiber layer (RNFL), has been damaged during the progression. Therefore, it is critical to investigate the original retinal structure parameters in subjects with PACS before injury changes initiate due to increased intraocular pressure (IOP).

Anterior segment anatomy in subjects with PACS has been extensively studied. Shallow anterior chamber depth (ACD), small cornea, thick lens thickness (LT), and short axial length (AL) [8-10] are found to be the risk factors for angle closure, which are significantly different from normal controls. Nevertheless, few research studies have explored the aspects of the retina and optic nerve in subjects with PACS, although they are targets of glaucomatous damage. The RGC is the preferred site of glaucomatous optic neuropathy. Ganglion cell complex (GCC) thickness is widely recognized as a better indicator for early detection of glaucoma with its high sensitivity and specificity $[11,12]$. Commercially available GCC normative database is mainly from normal control population. Given that, the present study aimed to investigate and compare the GCC parameters in subjects with and without PACS and further explore factors associated with the GCC difference.

\section{Methods}

Participants aged over 50 years in a rural area of Daxing District, Beijing, were invited to receive a screening examination to identify PACS and normal control subjects from October 2018 to November 2018. The study was approved by the Ethics Committee of Clinical Research in Beijing Tongren Hospital, Capital Medical University. The informed consent form was signed by all participants attending this process. This procedure was consistent with the principles of the Helsinki Declaration. The registration number in the Chinese Clinical Trial Registry is ChiCTR2000037944.

All subjects underwent the questionnaire survey and systemic and eye examination. We collected data of age, gender, height, body weight, waist, glucose, heart rate, diastolic blood pressure (DBP), systolic blood pressure (SBP), best-corrected visual acuity (BCVA), spherical refractive error (SER), IOP measured by using a noncontact tonometer without anesthesia (Topcon CT-80; Topcon Medical Systems, Inc., Oakland, NJ, USA), AL, ACD, LT, central corneal thickness (CCT) measured by using Lenstar (Lenstar LS900; Haag Streit, Bern, Switzerland), and RNFL and GCC thickness (Optovue; Optovue Inc., CA, USA). The IOP and ocular biometry parameters were measured 3 times, and the average value was taken for statistical analysis. At the end of the study, the gonioscopy examination was performed by experienced glaucoma doctors (L.S.N. and H.Y.) under topical anesthesia with a gonioscope (Ocular; Ocular Technology Inc., CA, USA) in a dark room to obtain detailed information of the anterior chamber angle. The static gonioscopy was performed first with 1-mm light beam away from the pupil in the primary position of gaze, and the dynamic gonioscopy was performed later with indentation and increased illumination. The retinal thickness was obtained from the $80-\mathrm{kHz}$ RTVue XR OCT with AngioVue software 2017.1 version. All patients avoided the eyelid obstruction, and their retina was scanned by the angio-OCT while glaring at the fixation lamp. The scanning was performed by a trained operator and read by an experienced ophthalmologist masked to other clinical examinations.

The diagnostic criteria for PACS were (1) pigmented trabecular meshwork invisible $>180^{\circ}$ in the primary position under static gonioscopy in the dark room and (2) normal IOP and absence of peripheral anterior synechiae. The inclusion criteria for normal controls were almost identical to PACS, except for the open anterior chamber angle under gonioscopy. The exclusion criteria of all participants included glaucoma, uveitis, iris neovascularization, macular diseases, eye trauma, ocular tumor, severe ocular media opacity, SER $>+3.00$ or $<-6.00 \mathrm{dpt}$, and any history of previous intraocular procedures. If both eyes met the inclusion criteria, only the right eye will be selected for the research. The exclusion criteria for the image quality of angio-OCT were (1) signal strength index below 60 and (2) severe movement artifacts.

The retina images provided the full retina thickness (from ILM to retina pigmented epithelium), GCC thickness or inner retinal thickness (from ILM to inner plexiform layer), and outer retina thickness (from inner plexiform layer to retina pigmented epithelium). All 3 layers were divided into the superior hemisphere and inferior hemisphere (GCC_Average, GCC_Superior_Avg, and GCC_Inferior_Avg). It was a 6-mm diameter ring centered $1 \mathrm{~mm}$ temporal to the fovea to emphasize the temporal retina region, which corresponds to the nasal visual field (area most susceptible to early glaucomatous damage). Ganglion cell complex global loss volume (GCC_GLV) represents the average amount of GCC loss in the fractional deviation map, which is calculated as the area of the pixels $<0$ divided by the total area. Ganglion cell complex focal loss volume (GCC_FLV) showed the percentage of the significant focal amount of GCC loss in the normalized standard pattern deviation map, which is calculated by dividing the area of the pixels $<0$ and $p<5 \%$ by the total area. The relationship of GCC_GLV and GCC_FLV is similar to that of mean deviation and pattern standard deviation in the visual field. Ganglion cell complex root mean square (GCC_RMS) showed the coefficient between the fractional or pattern deviation of an individual map and the normal pattern map. The better the maps match, the lower the value. GCC_GLV, GCC_FLV, and GCC_RMS were proved to change in the progress of glaucoma and show good ability in the diagnosis of glaucoma with the area under receiver operating characteristic curves ranging from 0.804 to 0.957 [12-14].

\section{Statistical Analysis}

The Shapiro test was used to check whether continuous variables were normally distributed. The median value and interquartile range were used for the basic statistical description of nonnormally distributed continuous variables. Frequency and percentage were used for basic statistical descriptions of categorical variables. The Wilcoxon rank-sum test was used to make the comparison of nonnormally distributed continuous variables between the PACS subgroup and the normal control subgroup; the $\chi^{2}$ test was used to make comparison of categorical variables between the PACS subgroup and the normal control subgroup. A linear mixed-effects model was used to explore the influence factors for GCC. The significance level was set to be 0.05 , 2-tailed. 
Table 1. Demographic data of enrolled subjects in PACS and normal controls

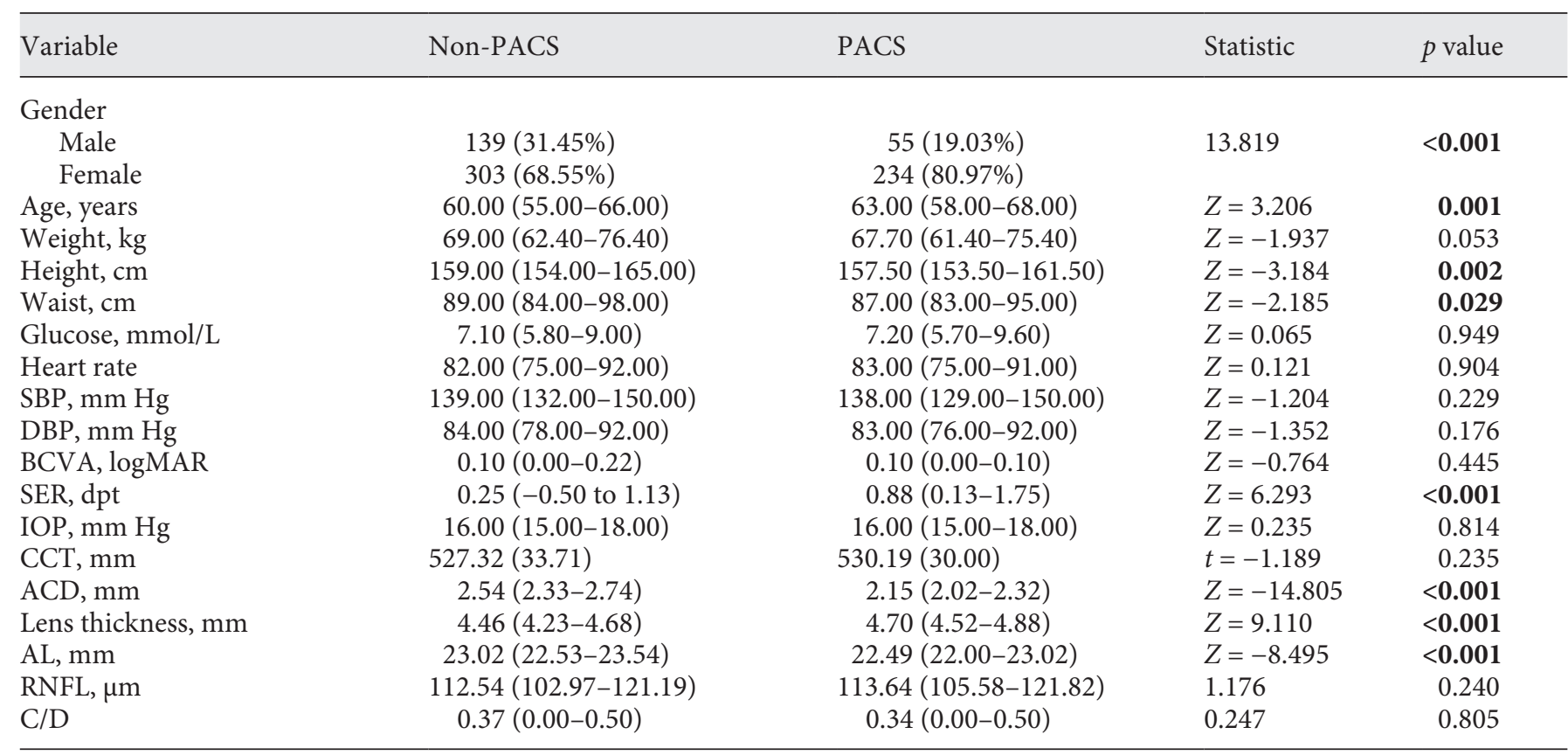

PACS, primary angle closure suspect; SBP, systolic blood pressure; DBP, diastolic blood pressure; BCVA, best-corrected visual acuity; SER, spherical refractive error; IOP, intraocular pressure; CCT, central corneal thickness; ACD, anterior chamber depth; AL, axial length; RNFL, retinal nerve fiber layer; C/D, cup/disk ratio.

Table 2. Comparison of macular parameters between PACS and normal controls

\begin{tabular}{|c|c|c|c|c|c|}
\hline Average SSI & $70.34(61.62-75.26)$ & $69.54(62.09-75.65)$ & $Z=0.116$ & 0.908 & 0.908 \\
\hline GCC/inner retina average, $\mu \mathrm{m}$ & $96.72(92.48-101.29)$ & $95.63(90.73-100.53)$ & $Z=-1.973$ & 0.049 & 0.147 \\
\hline GCC superior average, $\mu \mathrm{m}$ & $96.56(91.62-101.76)$ & $95.31(89.57-100.67)$ & $Z=-2.425$ & 0.015 & 0.045 \\
\hline GCC inferior average, $\mu \mathrm{m}$ & $96.96(93.14-101.41)$ & $96.08(91.32-100.86)$ & $Z=-1.751$ & 0.080 & 0.240 \\
\hline GCC S-I average, $\mu \mathrm{m}$ & $-0.14(-2.30$ to 2.11$)$ & $-0.68(-2.75$ to 1.33$)$ & $Z=-2.076$ & 0.038 & 0.038 \\
\hline GCC_RMS, \% & $0.08(0.07-0.10)$ & $0.08(0.07-0.11)$ & $Z=3.190$ & 0.001 & 0.001 \\
\hline Full retina average, $\mu \mathrm{m}$ & $269.93(262.07-278.34)$ & $268.87(260.47-275.70)$ & $Z=-1.683$ & 0.093 & 0.279 \\
\hline Full retina superior average, $\mu \mathrm{m}$ & $272.28(264.48-280.82)$ & $270.42(261.67-278.39)$ & $Z=-1.939$ & 0.053 & 0.159 \\
\hline Full retina inferior average, $\mu \mathrm{m}$ & $268.00(260.19-276.12)$ & $267.05(258.54-273.61)$ & $Z=-1.571$ & 0.116 & 0.348 \\
\hline Full retina $S-I$ average, $\mu \mathrm{m}$ & $4.83(1.50-8.15)$ & $4.57(1.27-7.17)$ & $Z=-1.101$ & 0.271 & 0.271 \\
\hline Outer retina average, $\mu \mathrm{m}$ & $172.92(168.62-178.25)$ & $173.19(168.44-177.61)$ & $Z=-0.734$ & 0.463 & 0.999 \\
\hline
\end{tabular}

PACS, primary angle closure suspect; SSI, signal strength index; GCC, ganglion cell complex; S-I, mean difference between superior and inferior; GCC_FLV, ganglion cell complex focal loss volume; GCC_GLV, ganglion cell complex global loss volume; GCC_RMS, ganglion cell complex root mean square. $p$ value was adjusted using the Bonferroni method. 
Table 3. Influence factors for GCC in PACS

\begin{tabular}{|c|c|c|c|c|c|c|}
\hline & \multicolumn{3}{|c|}{ Univariate analyses } & \multicolumn{3}{|c|}{ Multivariate analyses } \\
\hline & $\beta$ & $t$ & $p$ value & $\beta$ & $t$ & $p$ value \\
\hline Gender & 3.086 & 1.300 & 0.212 & & & \\
\hline Age (years) & -0.352 & -4.350 & $<0.001$ & -0.234 & -2.680 & 0.008 \\
\hline Weight (kg) & -0.010 & -0.190 & 0.848 & & & \\
\hline Height $(\mathrm{cm})$ & 0.051 & 0.630 & 0.529 & & & \\
\hline Waist $(\mathrm{cm})$ & -0.038 & -0.710 & 0.481 & & & \\
\hline Glucose (mmol/L) & -0.536 & -3.230 & 0.001 & -0.546 & -3.290 & 0.001 \\
\hline Heart rate & -0.063 & -1.550 & 0.123 & & & \\
\hline SBP (mm Hg) & -0.074 & -2.300 & 0.022 & -0.004 & -0.120 & 0.902 \\
\hline DBP (mm Hg) & -0.075 & -1.810 & 0.071 & & & \\
\hline BCVA (logMAR) & -9.383 & -1.240 & 0.219 & & & \\
\hline SER (dpt) & -0.373 & -2.060 & 0.041 & -0.291 & -0.850 & 0.395 \\
\hline IOP (mm Hg) & -0.477 & -1.240 & 0.219 & & & \\
\hline $\mathrm{CCT}(\mathrm{mm})$ & -0.013 & -0.750 & 0.455 & & & \\
\hline $\mathrm{ACD}(\mathrm{mm})$ & -0.166 & -0.080 & 0.935 & & & \\
\hline Lens thickness (mm) & 0.375 & 0.220 & 0.829 & & & \\
\hline $\mathrm{AL}(\mathrm{mm})$ & -0.071 & -0.100 & 0.921 & & & \\
\hline
\end{tabular}

GCC, ganglion cell complex; PACS, primary angle closure suspect; SBP, systolic blood pressure; DBP, diastolic blood pressure; BCVA, best-corrected visual acuity; SER, spherical refractive error; IOP, intraocular pressure; CCT, central corneal thickness; ACD, anterior chamber depth; AL, axial length.

For multiple comparisons of the same parameter, the $p$ value was adjusted using the Bonferroni method. All the analyses were done using an open source R program (https://www.r-project. org/, Version 4.0.2).

\section{Results}

A total of 1,419 subjects were examined; 821 subjects received gonioscopy examination, and among them, 731 eligible subjects were enrolled in our study: 289 subjects are PACS and 442 subjects are normal controls. The demographic and basic clinical information of the 2 groups is shown in Table 1. There were no significant differences in BCVA, heart rate, SBP, DBP, body weight, glucose, IOP, CCT, RNFL, cup to disc ratio, inferior GCC thickness, full retina thickness, and outer retina thickness between the 2 groups $(p>0.05)$. However, significant differences were found in gender, age, SER, height, waist, ACD, LT, AL, average GCC thickness, GCC_FLV, GCC_ GLV, and GCC_RMS between PACS and normal controls. For the systematical parameters, the PACS group had more females $(p<0.001)$, older ages $(p<0.001)$, lower body height $(p=0.002)$, and smaller waist circumference $(p=0.029)$. For the ocular structure of the anterior chamber, the PACS group displayed shallower ACD $(p<$
$0.001)$, thicker LT $(p<0.001)$, and shorter AL $(p<0.001)$. Higher SER $(p<0.001)$ was also found in the PACS group.

GCC_FLV, GCC_GLV, and GCC_RMS values were significantly larger in the PACS group compared to the normal control group $(p<0.001, p=0.007$, and $p=0.001$, respectively). In addition, the PACS group had less superior GCC thickness ( $p=0.045)$ than that in subjects without PACS. There were no significant differences in average GCC thickness and inferior GCC thickness, full retina thickness, and outer retina thickness between the 2 groups (Table 2).

To determine the correlation of risk factors associated with GCC average thickness in PACS and normal control groups, the linear mixed-effects model was conducted, respectively. For PACS patients, 5 variables (age, SER, SBP, DBP, and blood glucose) were significantly correlated with GCC thickness (Table 3). Moreover, multiple linear regression showed age $(p=0.008)$ and blood glucose $(p=0.001)$ were negatively correlated with GCC average thickness (Table 3). Based on the results, GCC thickness would decrease $0.234 \mu \mathrm{m}$ per year as the age of PACS patients increased, and GCC thickness would decrease $0.546 \mu \mathrm{m}$ for every $1 \mathrm{mmol} / \mathrm{L}$ as the blood glucose increased. For the normal control group, the linear mixedeffects model showed that age was the only significant 
Table 4. Influence factors for GCC in all subjects

\begin{tabular}{|c|c|c|c|c|c|c|}
\hline & \multicolumn{3}{|c|}{ Univariate analyses } & \multicolumn{3}{|c|}{ Multivariate analyses } \\
\hline & $\beta$ & $t$ & $p$ value & $\beta$ & $t$ & $p$ value \\
\hline PACS (yes vs. no) & -1.664 & -1.980 & 0.048 & -1.971 & -2.100 & 0.036 \\
\hline Gender & 0.053 & 0.060 & 0.955 & & & \\
\hline Age (years) & -0.299 & -5.020 & $<0.001$ & -0.258 & -3.760 & $<0.001$ \\
\hline Weight (kg) & 0.030 & 0.780 & 0.436 & & & \\
\hline Height $(\mathrm{cm})$ & 0.104 & 1.850 & 0.065 & & & \\
\hline Waist $(\mathrm{cm})$ & -0.006 & -0.150 & 0.880 & & & \\
\hline Glucose (mmol/L) & 0.009 & 0.070 & 0.943 & & & \\
\hline Heart rate & -0.001 & -0.040 & 0.965 & & & \\
\hline $\mathrm{SBP}(\mathrm{mm} \mathrm{Hg})$ & -0.033 & -2.400 & 0.020 & -0.004 & -0.140 & 0.893 \\
\hline DBP (mm Hg) & -0.046 & -1.940 & 0.049 & & & \\
\hline BCVA (logMAR) & -6.584 & -1.690 & 0.093 & & & \\
\hline $\operatorname{SER}(d p t)$ & -0.059 & 2.820 & 0.005 & 0.430 & 1.470 & 0.141 \\
\hline IOP (mm Hg) & -0.460 & -2.910 & 0.004 & 0.050 & 0.380 & 0.704 \\
\hline $\mathrm{CCT}(\mathrm{mm})$ & -0.012 & -1.010 & 0.315 & & & \\
\hline $\mathrm{ACD}(\mathrm{mm})$ & 2.838 & 1.400 & 0.161 & & & \\
\hline Lens thickness (mm) & -1.563 & -1.650 & 0.100 & & & \\
\hline $\mathrm{AL}(\mathrm{mm})$ & -0.518 & -1.220 & 0.223 & & & \\
\hline
\end{tabular}

GCC, ganglion cell complex; SBP, systolic blood pressure; DBP, diastolic blood pressure; BCVA, best-corrected visual acuity; SER, spherical refractive error; IOP, intraocular pressure; CCT, central corneal thickness; ACD, anterior chamber depth; AL, axial length.

influencing factor negatively correlated with average GCC thickness.

To identify all the factors associated with average GCC thickness, all subjects including PACS and normal controls groups were analyzed together. PACS, age, SER, SBP, DBP, and IOP were significantly associated with average GCC thickness by the linear mixed-effects model (Table 4$)$. Among them, PACS $(p=0.036)$ and age $(p<$ 0.001 ) were the key influencing factors for average GCC thickness in the linear mixed-effects model (Table 4). The GCC thickness in PACS was $1.97 \mu \mathrm{m}$ thinner than normal controls, and GCC thickness would decrease $0.258 \mu \mathrm{m}$ per year as the age increased.

\section{Discussion}

Macular RGC parameters are an important index for diagnosis and follow-up of glaucoma injury with the OCT technology progression from time-domain to spectraldomain [15-17]. A meta-analysis from AAO showed that macular RGC parameters have the same important role on glaucoma diagnosis comparing with RNFL measurement [18]. As one of the macular parameters, previous studies have documented that GCC has good sensitivity and specificity for diagnosis of glaucoma, and GCC measurement may be more useful for glaucoma patients with myopia $[11,12]$. However, it has been reported that a limited normative database of macular RGC parameters may influence the accuracy of diagnosis ability [19].

The normative database of GCC includes 656 eyes of 364 subjects, covering age from 18 to 70 years, 7 ethnicities, and refractive error from $-7.75 \mathrm{dpt}$ to $+5.50 \mathrm{dpt}$; the influence factors on measurements were age, optic disc size, and gender. Although it has been clinically widely used, the small sample size of the normative database limits its clinical evaluation and may induce misjudge of the results. For example, the inverse relationship between $\mathrm{AL}$ and GCC has been reported, the longer the AL, the thinner the GCC, which implied a broader range of AL might be needed in the normative database [20].

PACS is the stage with no glaucomatous change in the optic nerve and retina according to the definition [3]. Since PACS would progress to PACG, discovery of early PACG retinal damage should be more precise if comparing with a specific macula database of PACS than that with the now commercially available macular database because of its lack of PACS data, which may induce false decision. In the present cohort study, we first provided the parameters of GCC in PACS subjects. 
One important finding in our study was thinner superior GCC thickness and larger FLV and GLV in PACS than that in normal controls, which indicates that the normative database of GCC thickness in PACS was different compared with that in normal controls. The clinical meaning of this finding is that if we use the commercial GCC database as the baseline to evaluate the GCC parameters of PAC or PACG, it might overestimate the glaucomatous damage and result in false-positive diagnosis, especially that the GCC-FLV had been proven to be the most accurate predictor of visual field progression other than optic disc parameters [21-23]. The GCC database of PACS should be used as the baseline when evaluating PACD GCC change. Likewise, in high-myopia patients, a high myopic-specific GCC normal database had already been used to differentiate the glaucomatous damage for patients [24]. The finding in this study might offer a more accurate baseline database in reflection of the glaucomatous RGC loss in PACD.

There also might be another possible explanation for the thinner superior GCC and larger FLV and GLV in PACS subjects, which may due to the very early glaucoma changes of the fundus. However, the mean cup to disc ratio of PACS in our study is only 0.34 (ranging from 0.00 to 0.50 ), and the comparison of C/D and RNFL between PACS and normal controls in this study had no statistical significance; these results provided circumstantial evidence to support that the likelihood of GCC parameter changes due to glaucoma was slim. Further follow-up results may offer more stronger evidence.

Age and blood glucose were correlated with GCC thickness in PACS in our study. Longitudinal analysis showed the overall GCC thickness significantly decreased at an average rate of $0.25 \mu \mathrm{m} /$ year across all age groups [25], which is consistent with our results $(0.23-0.26 \mu \mathrm{m} /$ year $)$. The correlation of blood glucose with GCC thickness implied that high glucose might be a risk factor for evaluating GCC in subjects with PACS. Importantly, this finding indicated that correction of age and blood glucose might be necessary when evaluating GCC alterations in PACD.

There are several limitations to our study. First, all enrolled subjects are of Chinese ethnicity, so extrapolating to other races needs caution. Second, cross-section results may not provide solid evidence for the reasons of thin GCC and large FLV and GLV. Future follow-up examination should be considered. Third, the age and AL are proved different between PACS and normal controls, which may influence the GCC thickness in both groups. Further studies should focus on the matching of age and AL.

\section{Conclusion}

Our study provided novel evidence for the different characteristics of GCC parameters in PACS and the correlation of blood glucose and age with GCC thickness in PACS. These findings might offer a more accurate baseline database for patients with PACD and indicate the correction of age and blood glucose when evaluating GCC alterations in patients with PACD.

\section{Acknowledgments}

The authors thank the workers from Zhangziying Hospital, Daxing District, Beijing, China, for assisting with the recruitment of subjects.

\section{Statement of Ethics}

The study was approved by the Ethics Committee of Clinical Research in Beijing Tongren Hospital, Capital Medical University (Approval No. TRECKY2019-071). The clinical registration number is ChiCTR2000037944 in the Chinese Clinical Trial Registry. The informed consent form to participate was signed by all participants attending this process.

\section{Conflict of Interest Statement}

The authors of this manuscript have no conflicts of interest to declare.

\section{Funding Sources}

The study was funded by the National Natural Science Foundation of China (Grant No. 81970797).

\section{Author Contributions}

Ying Han and Shuning Li contributed to the conception of the study; Hui Feng, Yue Yang, Zhongyin Hu, and Fancheng Yan performed the experiment; Kai Cao performed the data analyses; Yizhen Tang and Xiaohua Pan wrote the manuscript.

\section{Data Availability Statement}

The data are stored in the form of paper materials in the Epidata database, which are not disclosed. 


\section{References}

1 Quigley HA. Number of people with glaucoma worldwide. Br J Ophthalmol. 1996;80(5): 389-93.

2 Foster PJ, Johnson GJ. Glaucoma in China: how big is the problem? Br J Ophthalmol. 2001;85(11):1277-82.

3 Foster PJ, Buhrmann R, Quigley HA, Johnson GJ. The definition and classification of glaucoma in prevalence surveys. Br J Ophthalmol. 2002;86(2):238-42.

4 Thomas R, George R, Parikh R, Muliyil J, Jacob A. Five year risk of progression of primary angle closure suspects to primary angle closure: a population based study. Br J Ophthalmol. 2003;87(4):450-4.

5 Yan C, Han Y, Yu Y, Wang W, Lyu D, Tang $\mathrm{Y}$, et al. Effects of lens extraction versus laser peripheral iridotomy on anterior segment morphology in primary angle closure suspect. Graefes Arch Clin Exp Ophthalmol. 2019; 257(7):1473-80.

6 Jiang Y, Chang DS, Zhu H, Khawaja AP, Aung $\mathrm{T}$, Huang $\mathrm{S}$, et al. Longitudinal changes of angle configuration in primary angle-closure suspects: the Zhongshan Angle-Closure Prevention Trial. Ophthalmology. 2014;121(9): 1699-705.

7 He M, Jiang Y, Huang S, Chang DS, Munoz B, Aung T, et al. Laser peripheral iridotomy for the prevention of angle closure: a single-centre, randomised controlled trial. Lancet. 2019; 393(10181):1609-18.

8 Lowe RF. Aetiology of the anatomical basis for primary angle-closure glaucoma. Biometrical comparisons between normal eyes and eyes with primary angle-closure glaucoma. $\mathrm{Br}$ J Ophthalmol. 1970;54(3):161-9.

9 Alsbirk PH. Primary angle-closure glaucoma. Oculometry, epidemiology, and genetics in a high risk population. Acta Ophthalmol Suppl. 1976(127):5-31.

10 Sihota R, Lakshmaiah NC, Agarwal HC, Pandey RM, Titiyal JS. Ocular parameters in the subgroups of angle closure glaucoma. Clin Exp Ophthalmol. 2000;28(4):253-8.
11 McCann P, Hogg RE, Wright DM, McGuinness B, Young IS, Kee F, et al. Diagnostic accuracy of spectral-domain OCT circumpapillary, optic nerve head, and macular parameters in the detection of perimetric glaucoma. Ophthalmol Glaucoma. 2019 Sep-Oct;2(5): 336-45.

12 Shoji T, Sato H, Ishida M, Takeuchi M, Chihara E. Assessment of glaucomatous changes in subjects with high myopia using spectral domain optical coherence tomography. Invest Ophthalmol Vis Sci. 2011;52(2):1098102.

13 Rao HL, Zangwill LM, Weinreb RN, Sample PA, Alencar LM, Medeiros FA. Comparison of different spectral domain optical coherence tomography scanning areas for glaucoma diagnosis. Ophthalmology. 2010;117(9):16929.e1

$14 \mathrm{Xu} \mathrm{H}$, Zong Y, Zhai R, Kong X, Jiang C, Sun $\mathrm{X}$. Intereye and intraeye asymmetry analysis of retinal microvascular and neural structure parameters for diagnosis of primary open-angle glaucoma. Eye. 2019;33(10):1596-605

15 Leung CK, Chan WM, Yung WH, Ng AC, Woo J, Tsang MK, et al. Comparison of macular and peripapillary measurements for the detection of glaucoma: an optical coherence tomography study. Ophthalmology. 2005; 112(3):391-400.

16 Wang M, Hood DC, Cho JS, Ghadiali Q, De Moraes CG, De Moraes GV, et al. Measurement of local retinal ganglion cell layer thickness in patients with glaucoma using frequency-domain optical coherence tomography. Arch Ophthalmol. 2009;127(7):875-81.

17 Tan O, Chopra V, Lu AT, Schuman JS, Ishikawa $\mathrm{H}$, Wollstein $\mathrm{G}$, et al. Detection of macular ganglion cell loss in glaucoma by Fourier-domain optical coherence tomography. Ophthalmology. 2009;116(12):2305-14 e12.
18 Oddone F, Lucenteforte E, Michelessi M, Rizzo S, Donati S, Parravano M, et al. Macular versus retinal nerve fiber layer parameters for diagnosing manifest glaucoma: a systematic review of diagnostic accuracy studies. Ophthalmology. 2016;123(5):939-49.

19 Chen HS, Liu CH, Lu DW. Comparison of glaucoma diagnostic accuracy of macular ganglion cell complex thickness based on nonhighly myopic and highly myopic normative database. Taiwan J Ophthalmol. 2016 Jan-Mar;6(1):15-20.

20 Song WK, Lee SC, Lee ES, Kim CY, Kim SS. Macular thickness variations with sex, age, and axial length in healthy subjects: a spectral domain-optical coherence tomography study. Invest Ophthalmol Vis Sci. 2010;51(8): 3913-8

21 Zhang X, Parrish RK 2nd, Greenfield DS, Francis BA, Varma R, Schuman JS, et al. Predictive factors for the rate of visual field progression in the advanced imaging for glaucoma study. Am J Ophthalmol. 2019;202:62-71.

22 Zhang X, Dastiridou A, Francis BA, Tan O, Varma R, Greenfield DS, et al. Baseline Fourier-domain optical coherence tomography structural risk factors for visual field progression in the advanced imaging for glaucoma study. Am J Ophthalmol. 2016;172:94-103.

23 Zhang X, Loewen N, Tan O, Greenfield DS, Schuman JS, Varma R, et al. Predicting development of glaucomatous visual field conversion using baseline Fourier-domain optical coherence tomography. Am J Ophthalmol. 2016;163:29-37.

24 Wang WW, Wang HZ, Liu JR, Zhang XF, Li $\mathrm{M}$, Huo YJ, et al. Diagnostic ability of ganglion cell complex thickness to detect glaucoma in high myopia eyes by Fourier domain optical coherence tomography. Int J Ophthalmol. 2018;11(5):791-6.

25 Zhang X, Francis BA, Dastiridou A, Chopra $\mathrm{V}$, Tan O, Varma R, et al. Longitudinal and cross-sectional analyses of age effects on retinal nerve fiber layer and ganglion cell complex thickness by Fourier-domain OCT. Transl Vis Sci Technol. 2016;5(2):1. 